\title{
Research Paper \\ Depression, Anxiety and Suicidal Ideation Among Patients With Chronic Kidney Disease Undergoing Hemodialysis in Qazvin, Iran
}

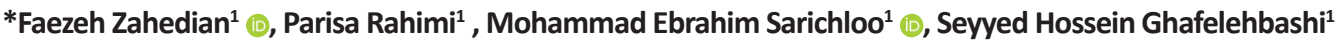

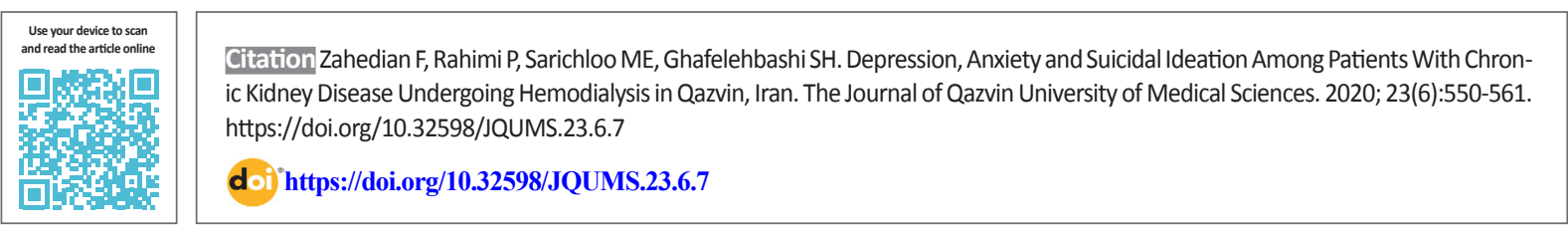

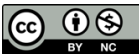

Received: 21 Sep 2019 Accepted: 24 Dec 2019 Available Online: 01 Feb 2020

Keywords: Depression, Anxiety, Suicidal ideation, Chronic kidney disease, Hemodialysis

\section{A B STRACT}

Background Patients undergoing hemodialysis are prone to psychiatric symptoms due to considerable changes in their individual, social and occupational life which can influence the course and prognosis of renal diseases.

Objective The aim of the present study is to evaluate the prevalence of depression, anxiety, and suicidal ideation among patients with chronic kidney disease undergoing hemodialysis in Qazvin, Iran.

Methods This descriptive cross- sectional study was performed on 120 patients with chronic kidney disease undergoing hemodialysis in Qazvin, Iran. Prior to study, their demographic and laboratory data were recorded. Dialysis adequacy was assessed using urea reduction ratio (URR) and KT/V formula. Data collection tools were Beck's Depression Inventory, Beck's Anxiety Inventory and Beck Scale for Suicidal Ideation. Collected data were analyzed using Pearson correlation test and t-test.

Findings The mean level of depression in patients was 13.72 where $79(65.8 \%)$ had depressive symptoms. The severity of symptoms was mild in 47 (39.2\%), moderate in 21 (17.5\%), and severe in 11 (9.2\%). Moreover, the mean level of anxiety was 9.73 where 16 (13.3\%) had anxiety symptoms. Of 120 patients, only $8(6.7 \%)$ had suicidal ideation. There was a significant negative association between dialysis adequacy and the severity of depression, anxiety and suicidal ideation. Anxiety symptoms were more frequent among unemployed patients, and the increase in age was associated with the increase in suicidal ideations $(P<0.05)$.

Conclusion Regarding the high prevalence of depressive symptoms and existence of anxiety symptoms in patients undergoing hemodialysis in Qazvin, assessment of these psychiatric disorders along with effective therapeutic interventions should be considered.

\section{Extended Abstract}

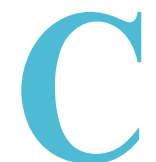

\section{Introduction}

hronic kidney disease is one of the major public health problems worldwide and is usually diagnosed as a silent disease in advanced stages; when hemodialysis and kidney transplantation are the only options $[1,2]$. Chronic physical diseases can affect the patients' mental health. In this regard, the prevalence of psychiatric symptoms in various chronic physical diseases has been considered in recent years $[4,6]$. Patients undergoing

\section{* Corresponding Author:}

Faezeh Zahedian

Address: Department of Psychiatry, Faculty of Medicine, Qazvin University of Medical Sciences, Qazvin, Iran

Tel: +98 (912) 7810264

E-Mail: fzahediyan@qums.ac.ir 
hemodialysis are prone to psychiatric disorders due to mobility limitations, stress of coping with disease, side effects of medications and comorbid diseases [7-10].

So far, a number of studies have investigated the prevalence of depression, anxiety, and suicidal ideation in patients undergoing hemodialysis. According to a meta-analysis, the overall prevalence of depression in hemodialysis patients in Iran is $63 \%$ [15]. The prevalence of depression is different in various parts of Iran. Najafi et al. in a study on 127 patients undergoing hemodialysis in Tehran found out that the prevalence of depression and anxiety among them were $31.5 \%$ and $41.7 \%$, respectively [16]. Due to the significant differences in the results of previously conducted studies and the limited number of studies conducted in this area in Qazvin province, the present study aimed to investigate the prevalence of depression, anxiety, and suicidal ideation in hemodialysis patients in Qazvin, Iran.

\section{Materials and Methods}

This study is a descriptive cross-sectional study conducted on 120 patients with chronic kidney disease undergoing hemodialysis in Booali Sina Hospital in Qazvin. The inclusion criteria were: Having age $>16$ years, minimum basic literacy, history of hemodialysis for at least 6 months, three times a week, each for four hours. Demographic information of all participants was recorded as well as their laboratory data including hemoglobin and creatinine levels which were collected from their medical records. Dialysis adequacy was calculated using urea reduction ratio (URR) and KT/V formula. In the next step, all patients were asked to complete beck's depression inventory (BDI), beck's anxiety inventory (BAI) and beck scale for suicidal ideation (BSSI). Collected data were analyzed using Pearson correlation test and T-test.

\section{Results}

Of 120 patients, 66 were males (55\%), 88.3\% married, and $11.6 \%$ single. The mean age of participants was $54.35 \pm 17.99$ years. The patients' mean BDI score was 13.72 , where $65.8 \%$ had depressive symptoms. The severity of symptoms was mild in $39.2 \%$, moderate in $17.5 \%$, and severe in $9.2 \%$. The mean BAI score of patients was reported 9.73 , where $13.3 \%$ of them had anxiety symptoms of which $12.5 \%$ had moderate anxiety, and only 1 had severe anxiety. The mean BSSI score of patients was 1.31, where only $6.7 \%$ were at high risk for suicide; no patients had very high risk for suicidal attempt. Dialysis adequacy was significantly correlated with severity of depression, anxiety, and suicidal ideation $(\mathrm{P}<0.05)$.

\section{Discussion}

The aim of this study was to determine the prevalence of depression, anxiety, and suicidal ideation in hemodialysis patients in Qazvin, Iran. The prevalence of depression was reported $65.8 \%$, which was mild to moderate in the most patients. There are different rates of the prevalence of depression reported in various studies in Iran. For example, a meta-analysis conducted by Mirzaei et al. on the prevalence of depression in patients undergoing hemodialysis from 1998 to 2013, showed that the overall prevalence of depression was between $50 \%$ and $70 \%$. In their study, the highest prevalence of depression was $93 \%$ in Sanandaj and the lowest prevalence rate was $28 \%$ in Tehran [15]. The difference in results observed in various studies can be due to differences in scales, sample size, study location, and socioeconomic status of patients. The overall results of all related studies, however, show a high prevalence of depressive symptoms in these patients [6].

The prevalence of anxiety in our study was $13.3 \%$, which was mild to moderate in most patients. Few studies have investigated the prevalence of anxiety in patients undergoing hemodialysis $[9,15,21]$. In one study using the Cattell Anxiety Inventory, Hajseyed Javadi et al. reported the prevalence of anxiety in Qazvin as 52\% [9].

In our study, only $6.7 \%$ of patients had suicidal thoughts. The relatively low prevalence of suicidal ideation in current study is consistent with the severity of mild-to-moderate depression in patients. Few studies have investigated suicidal ideation individually in patients undergoing hemodialysis. In a study on 180 patients, its prevalence in Sari City was reported $10.5 \%$ [23].

In our study, higher dialysis adequacy was associated with lower scores of depression, anxiety, and suicidal ideation. Most studies have examined the relationship between dialysis adequacy and quality of life and mortality rate; however, few studies have evaluated the association of dialysis adequacy with depression and anxiety [16, 27].

Najafi et al. showed no significant relationship among the dialysis adequacy, depression and anxiety, but Hashemi eta al. in a study on 46 hemodialysis patients in Shirvan City, found a significant negative relationship between dialysis adequacy and depression scores [27].

The significant negative relationship of dialysis adequacy with depression, anxiety, and suicidal ideation in our study may indicate a link between dialysis adequacy and mental health; it is likely that patients with adequate dialysis may have decreased disease symptoms. They can perform better 
and are therefore less likely to experience depression and anxiety [27].

The results of this study revealed the high prevalence of depressive symptoms and the existence of anxiety symptoms in hemodialysis patients in Qazvin. Evaluation of psychiatric symptoms, especially depression and anxiety should be considered in these patients along with therapeutic interventions if necessary.

\section{Ethical Considerations}

Compliance with ethical guidelines

This study was approved by the Research Ethics Committee of Qazvin University of Medical Sciences (Code: IR.QUMS.REC.1396.95). Informed consents were obtained from all the participants.

\section{Funding}

This study was extracted from the MS. thesis of second author approved by the Department of Psychiatry at Qazvin University of Medical Sciences.

\section{Authors' contributions}

Methodology: Seyyed Hossein Ghafelehbashi and Mohammad Ebrahim Sarichloo; Data analysis: Faezah Zahedian; Resources and initial draft preparation: Parisa Rahimi; Editing \& Review, and Project administration: Faezah Zahedian.

\section{Conflicts of interest}

The authors declared no conflict of interest.

Acknowledgements

The authors would like to thanks the Deputy for Research of Qazvin University of Medical Sciences. 


\title{
بررسى افسردتى، اضطراب و افكار خودكشى در بيماران مزمن كليوى تحت همودياليز
}

\author{
•فائزه زاهديان' نه بريسا رحيمى'، محمد ابراهيم ساريجلو' ه، سيد حسين قافلهباشى' \\ ا. كروه روانيزشكى، دائكده يزشكى، دانشكاه علوميزشكى قزوين، قزوين، ايران.
}

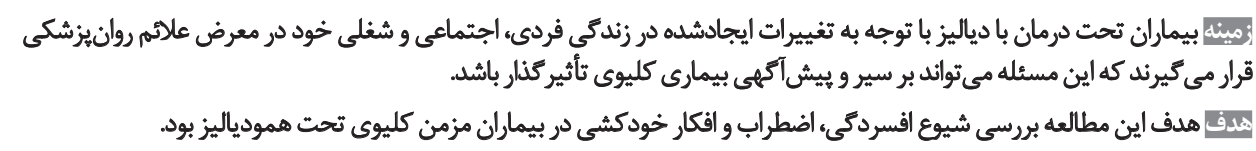

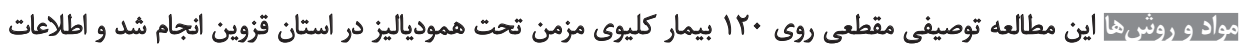

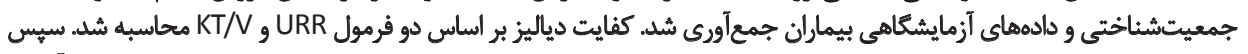

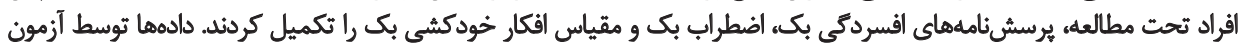

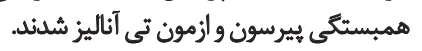

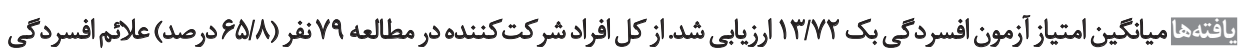

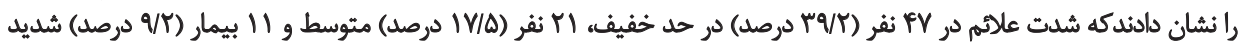

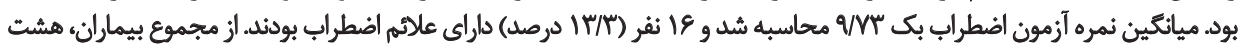

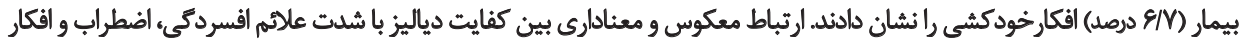

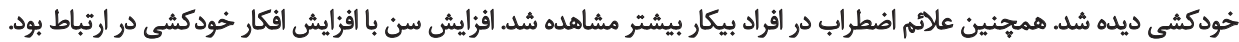

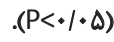

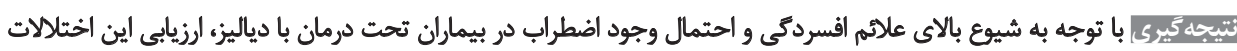

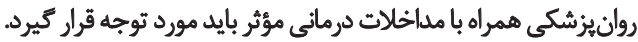

تاريخ دريافت: • ش شهريور تاريخ بذيرش: با•دى

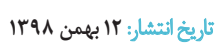

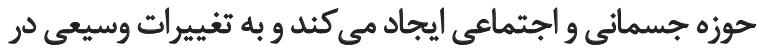

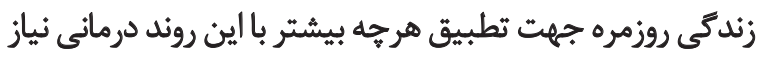

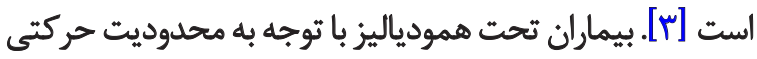

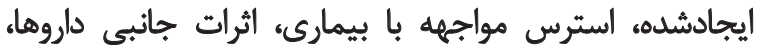

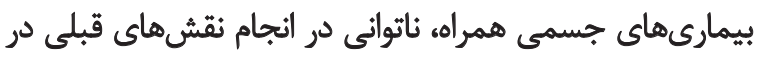

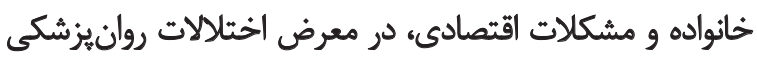

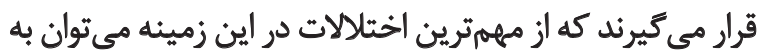

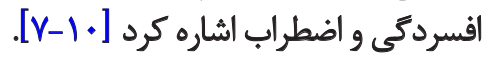

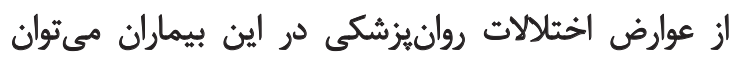

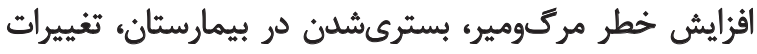

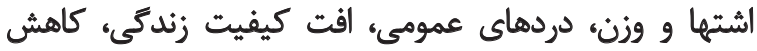

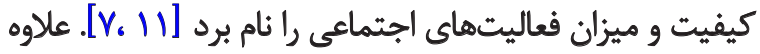
بر اين موارد، افسردگى و اضطراب روى رئيت نتيجه درمانى بيماران

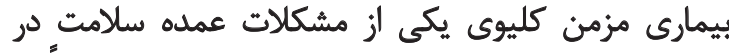

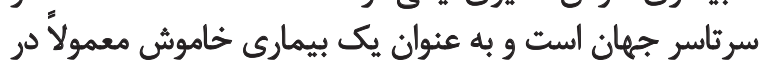

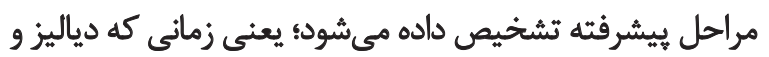

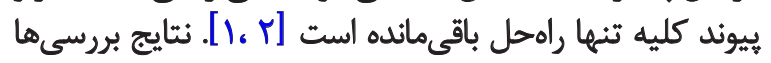

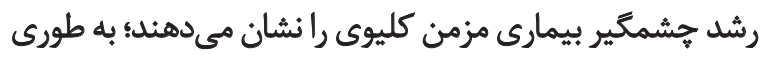

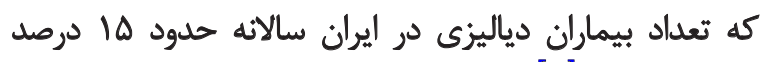

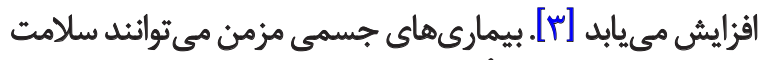

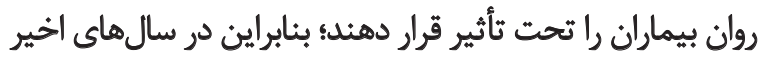

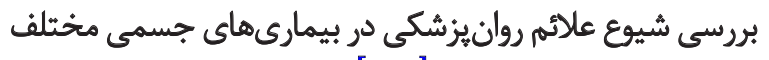

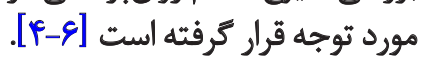

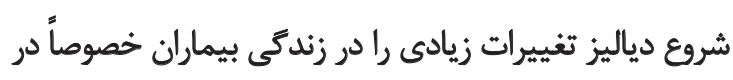


به ارتباط مثفاوت بين عوامل فردى و اجتماعى با شيوع علاثم

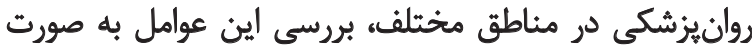

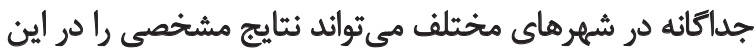
زمينه در هر منطقه در اختيار قرار دهد.

\section{مواد و روش مها}

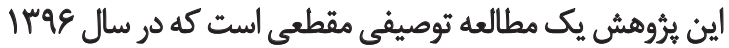

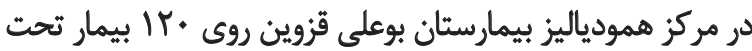

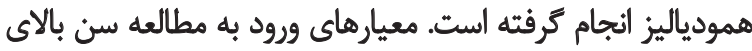

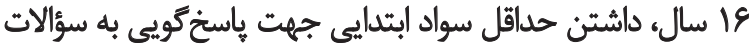

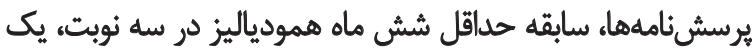

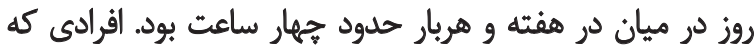

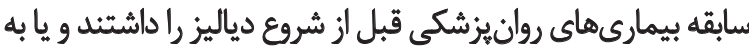

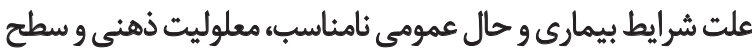

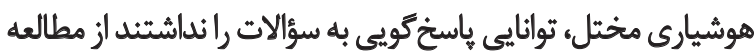

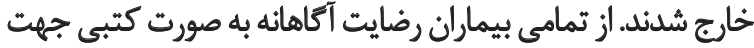

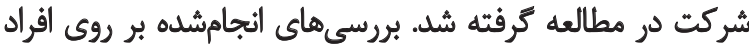

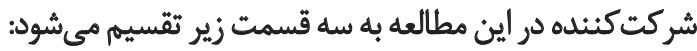
ا. در مرحله اول اطلاعات جمعيتشناختى شامل سن، جنسيت،

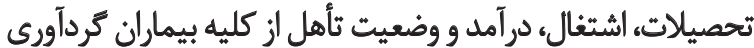

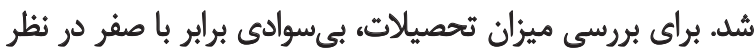

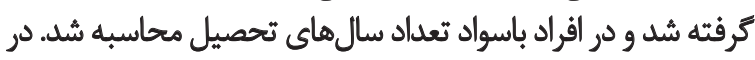

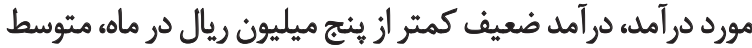

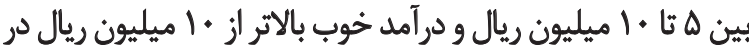

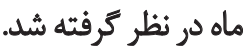

r. اطلاعات آزمايشكاهى شامل هموكلوبين و كراتينين از يرونده

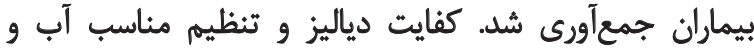

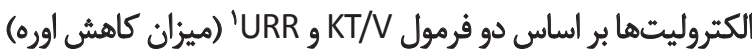

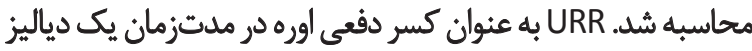
است كه بر اساس فرمول شماره المحاسبه شد:

ميزان كاهش/وره =(اوره بعلد دياليزث/ورهقبل ازدياليز) - (x×. روش ترجيحى ارزيابى كفايت دياليز است و بر اساس

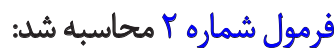

$K T / V=(K \times T) \div(V)$

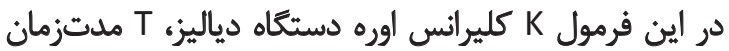

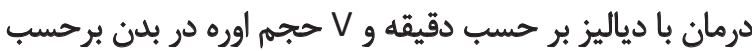

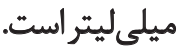

1. Urea reduction rate
كليوى تأثير مى كذارد. شروع اين اختلالات با هذيرش درمانى

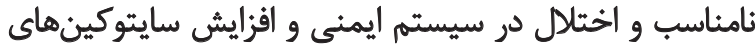

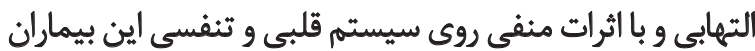

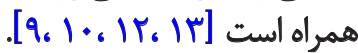

على رغم اين تفاسير، اشتباه در تشخيص اختلالات روانيزشكى

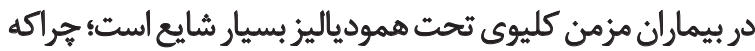

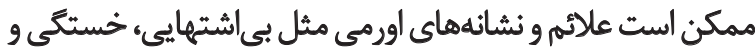

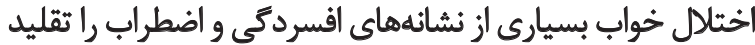

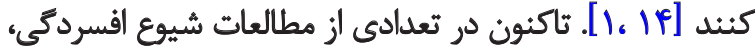

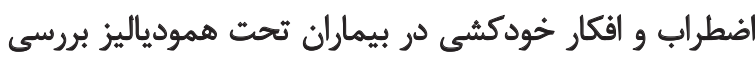

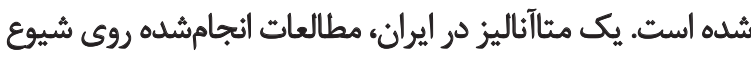

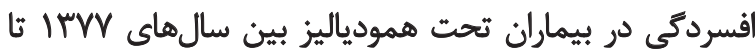

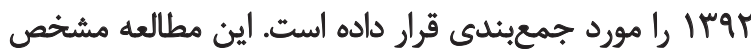

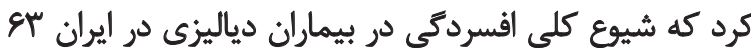

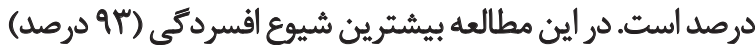

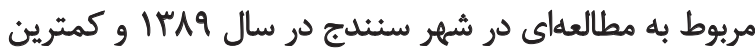

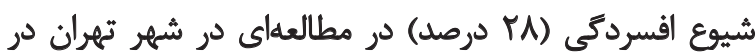

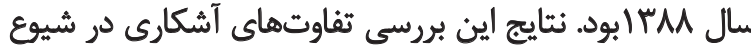

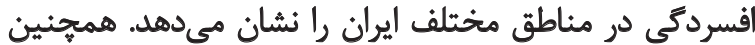

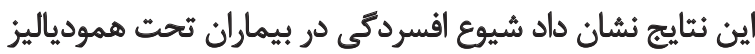

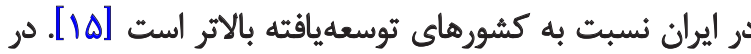

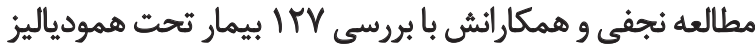

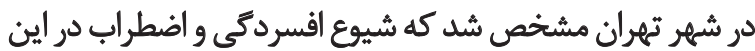

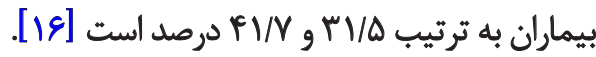

با توجه به تفاوتهاى قابل توجه و برجسته در نتايج اين

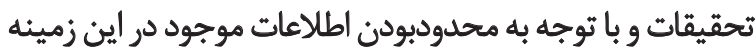

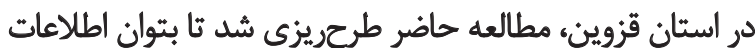

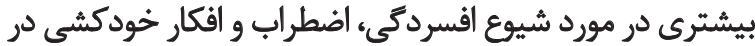

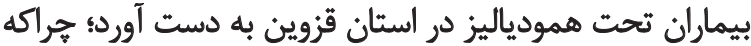

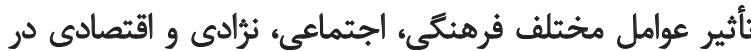

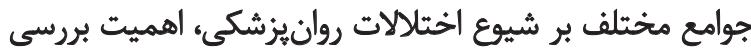

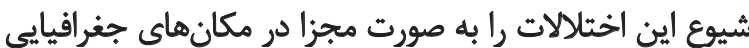

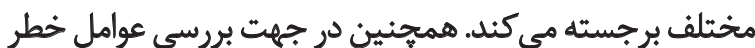

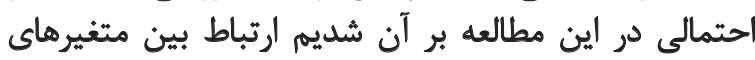

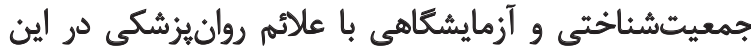

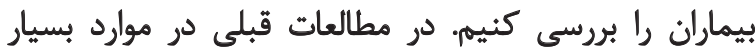

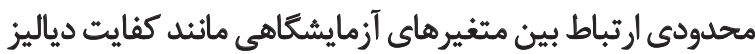

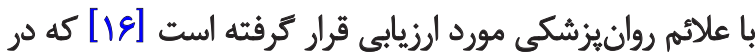

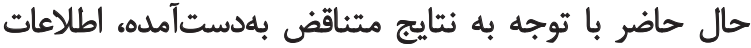

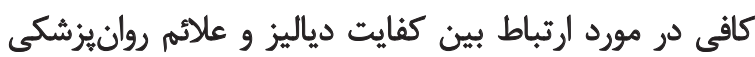

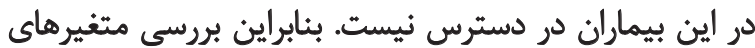

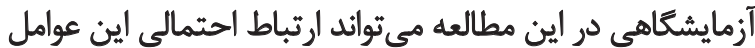

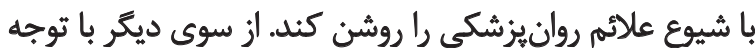


متغيرهاي كيفى توسط آزمون تى بررسى شد. سطح معنى دارى

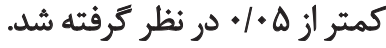

Lيافتهان

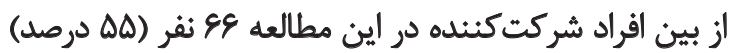

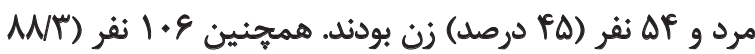

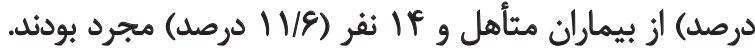

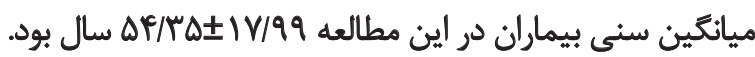

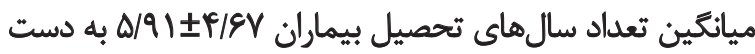

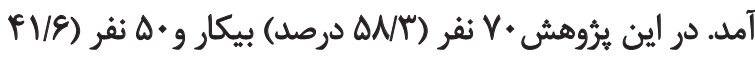

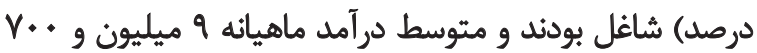

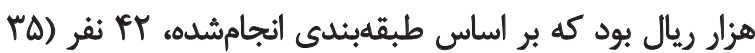

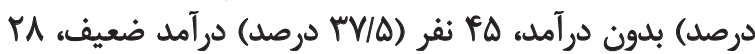

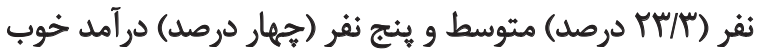

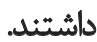

با بررسى دادهاي آزمايشكاهى ميانكين آخرين آزمايش

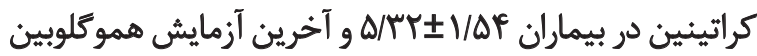
IVAF士 V/AF

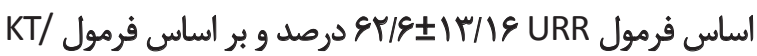

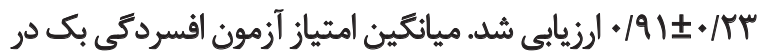

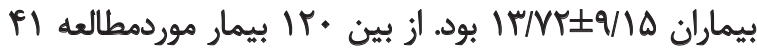

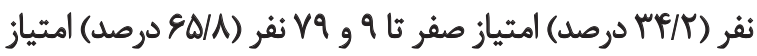

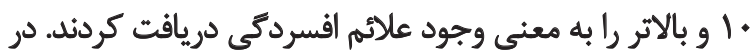

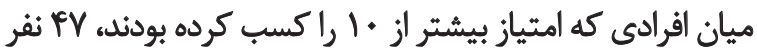

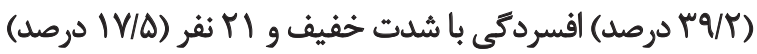

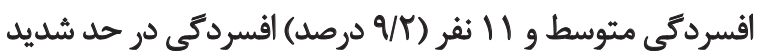

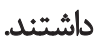

در بيماران موردمطالعه ميانكين نمره كسبشده در آزمون

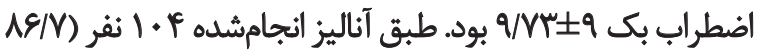

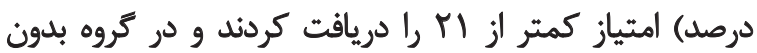

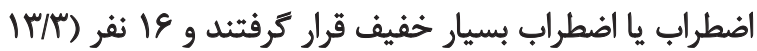

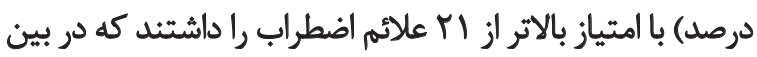

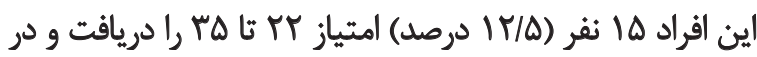

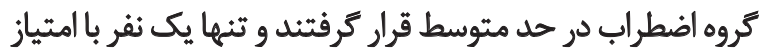

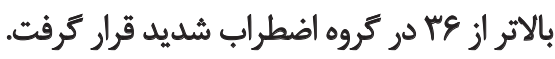

ميانكين امتياز افكار خودكشى در جمعيت موردمطالعه

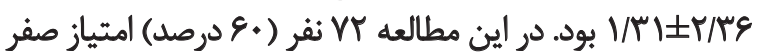

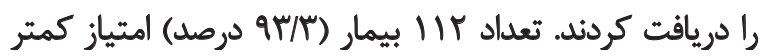

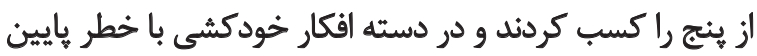

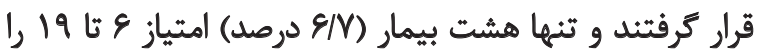

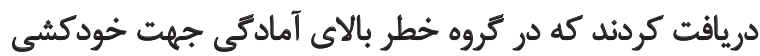

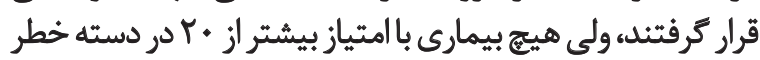
بسيار بالاي اقدام به خودكشى قرار نئرفت.

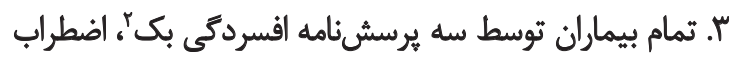

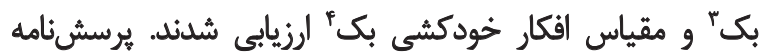

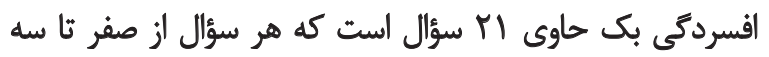

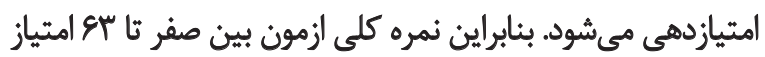

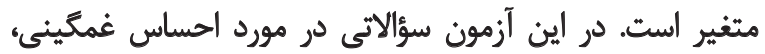

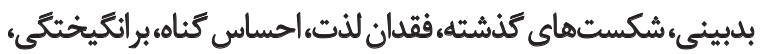

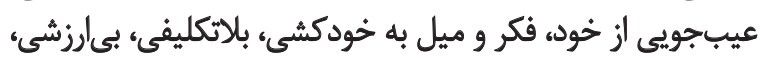

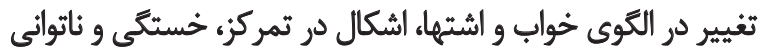

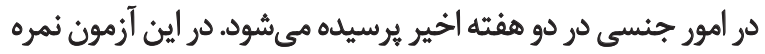

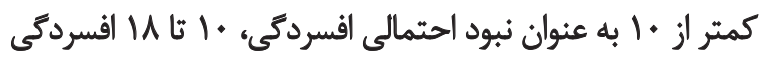

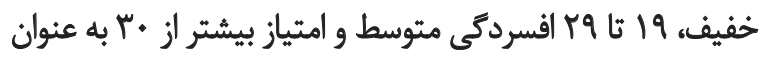

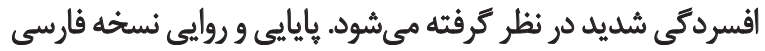

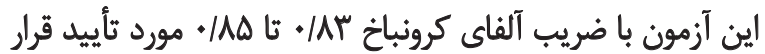

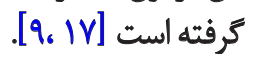

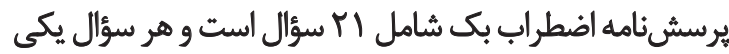

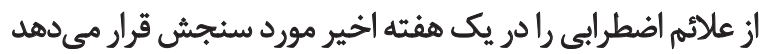

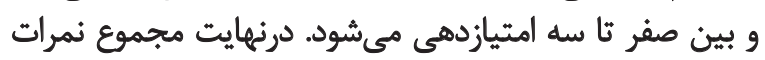

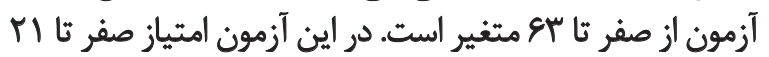

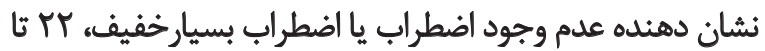

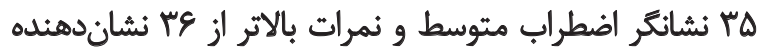

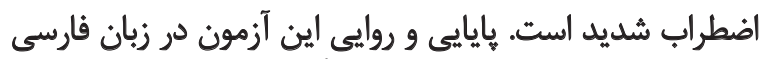

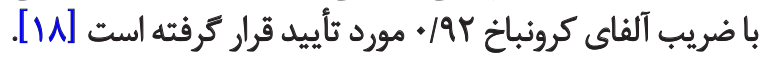

مقياس افكار خودكشى بك شامل 19 سؤال است و مواردى

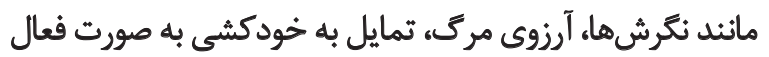

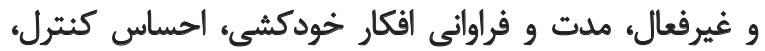

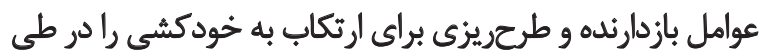

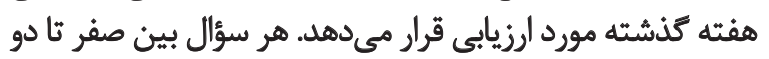

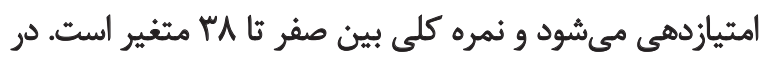

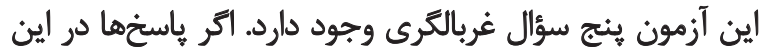

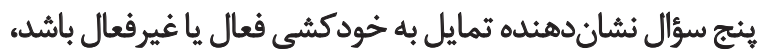

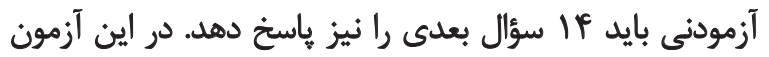

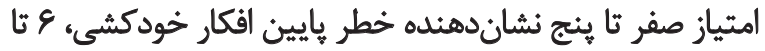

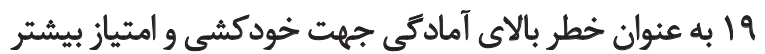

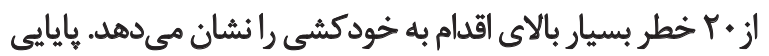

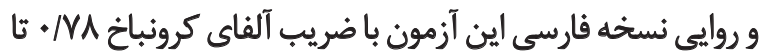

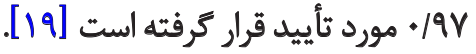

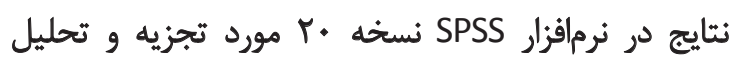

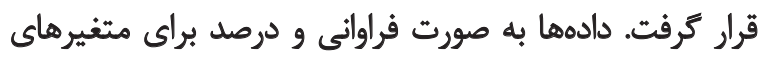

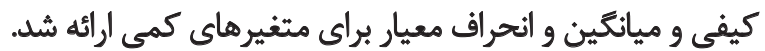

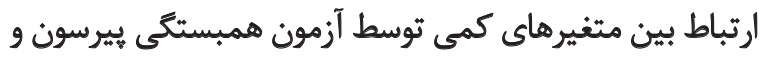

2. Beck's depression inventory

3. Beck's anxiety inventory

4. Beck scale for suicidal ideation 
جدول (ـ شيوع و شُت علائم افسردكى، اضطراب و افكار خودكشى به تفكيك جنسيت، درآمد، اشتغال و وضعيت تأهل

\begin{tabular}{|c|c|c|c|c|c|c|c|c|c|c|c|c|}
\hline \multicolumn{2}{|c|}{ تأهل } & \multicolumn{2}{|c|}{ أشتغال } & \multicolumn{2}{|c|}{ جنسيت } & \multicolumn{4}{|c|}{ درآمد } & \multirow{2}{*}{\multicolumn{2}{|c|}{ مثغير }} & \\
\hline مجرد & مثاهل & شياغل & بيكار & زن & مرد & حُوب & متوسط & ضعيف & بدون درآمد & & & \\
\hline f & $r$ & M & $M$ & r. & $m$ & r & $\pi$ & iv & 1. & تعلداد & بلدون افسردىى & \\
\hline$r / r$ & $r \cdot / \Lambda$ & 10 & $|9|$ & $18 / 8$ & $I V / \Delta$ & V/8 & 1. & $\| F / 1$ & Nr & درصد & المثياز صفر تأه & \\
\hline 8 & il & 19 & rA & $r$. & $r V$ & $r$ & 1. & 18 & 19 & تعلاد & افسردكى خفيف & \\
\hline$\Delta$ & $m \in /$ & $\mid \alpha / \Lambda$ & $\pi / \pi$ & $19 / 8$ & $T r / \Delta$ & Ve & $N{ }^{N}$ & 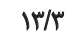 & $\mid \theta / A$ & درصد & $\mid$ & 可 \\
\hline$r$ & 19 & 9 & ir & $r$ & if & - & $r$ & 11 & $r$ & تعلداد & افسردكى متوسط & 3 \\
\hline$V / 8$ & $18 / A$ & $V / \Delta$ & 1. & $\Delta / \Lambda$ & $11 / 8$ & - & $r / \Delta$ & VI & $\Delta / \lambda$ & درصد & المثياز 19 ت5 & \\
\hline r & 9 & f & $v$ & $v$ & f & 1 & $r$ & 1 & 8 & تعلاد & افسردكي شديد & \\
\hline V/8 & $V / \Delta$ & $r / r$ & $\Delta / A$ & $\Delta / \Lambda$ & $r / r$ & .11 & $T / \Delta$ & $\cdot / A$ & $\Delta$ & لرصد & امتياز بيشتر از •+r & \\
\hline ir & ar & fe & $\Delta A$ & ro & $\Delta q$ & ه & $\pi$ & FT & $\pi$ & تعدلاد & اضطراب بسميارخفيف & \\
\hline$\Lambda \Delta, Y$ & $N \in \mathcal{A}$ & ar & Ar,q & $T V / \Delta$ & $p q /$ & $r / 1$ & r. & ro & $T V / \Delta$ & درصد & المثياز صفر تا ال & \\
\hline$r$ & Ir & r & 11 & $\wedge$ & $\checkmark$ & - & r & $r$ & $\Lambda$ & تعلاد & اضطراب متوسط & $\overline{3}$ \\
\hline $1 / 8$ & 1.11 & $r / \%$ & 2/ & $9 / 8$ & $\Delta / A$ & - & $r / r$ & $T / Q$ & $9 / 8$ & نرصد & المتياز بTتا & $\frac{2}{3}$ \\
\hline - & 1 & + & 1 & 1 & + & - & + & - & 1 & تعلاد & اضطراب شديد & \\
\hline - & $\cdot 11$ & - & $\cdot / A$ &.$/ 1$ & - & . & - & . & $\cdot / 1$ & درصد & امتياز بالاتر از وب & \\
\hline ir & $1 .$. & Po & or & ه) & \& & $\Delta$ & $m$ & $\mathrm{pr}$ & rq & تعلاد & خطر يايين & $\overline{2}$ \\
\hline 1. & $\Delta \mathrm{N} / \mathrm{N}$ & $r V / \Delta$ & $\Delta \triangle / A$ & $T r / \Delta$ & $0 . / \lambda$ & $M$ & r. & re/s & $\pi / \Delta$ & درصد & امثياز كمثر از هـ & 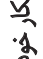 \\
\hline$r$ & 8 & $\Delta$ & $r$ & $r$ & $\Delta$ & - & $\varphi$ & 1 & $r$ & تعلاد & حُطر بالا & 棒 \\
\hline $1 / 8$ & $\Delta$ & $\% / 1$ & $r / \Delta$ & $r / \Delta$ & $4 / 1$ & - & $\mu / \pi$ &.$/ A$ & $T / \Delta$ & درصد & امثياز ع ثا 19 & \\
\hline
\end{tabular}

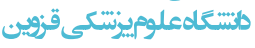

جدول r. ارتباط بين متغير هاى جمعيتشناختى و آزمايشكاهى با شدت علائم افسردىى، اضطراب و افكار خودكشى (سطح معنى دارى)

\begin{tabular}{|c|c|c|c|c|c|c|c|c|c|c|}
\hline KT/V & URR & هموتكلوبين & كراتينين & درأمد & اشتغال & تأهل & تحصيلات & جنسيت & سن & مثثير \\
\hline$* r^{*}$ & $.1 .0 \%$ &.$/ M I \Delta$ &.$/ M Y Y$ &.$/ F A$ & - Mas & - Nar & - lava & - MET & / MHI & افسرد5ى \\
\hline$* *+r^{*}$ & $.1 .+1^{\circ}$ &.$/ N F$ &.$/ F B$ & . /OYA & $\circ * \mu^{*}$ &.$/ 4 T$ & r &.$/ 191$ & - MIF & اضطراب \\
\hline$* /+\infty 1^{\circ}$ & $+1++4$ & +1948 & $* / M_{0}$ &.$/ F V A$ & . & $. / 1 / 90$ & RIT & $+/ \Lambda \Delta \varepsilon$ & $+1+\infty \phi^{*}$ & افكار خودكشي \\
\hline
\end{tabular}

همجنين بار امترهاى آزمايشكاهى هموكلوبين و كراتينين ارتباط معنادارى وجود نداشت (جدول شماريت أشماره (Y). s.s 5 dout gou

شيوع افسردكى در جمعيت اين مطالعه بر اساس آزمون

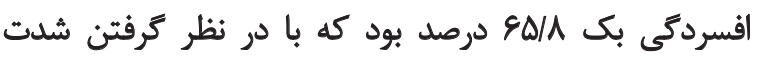
افسرد

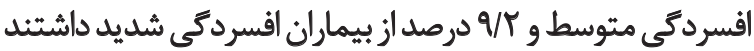

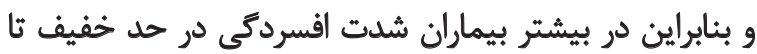

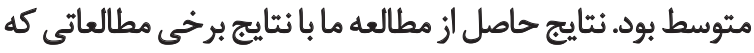
در ايران به بررسى شيوع افسردكى در بيماران ثتحت همودياليز

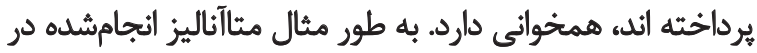
ايران توسط ميرزايى و همكارانش كه مطالعات انجامشده بين بين

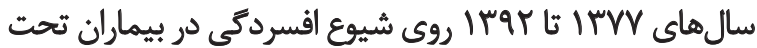
همودياليز را بررسى كردند، نشان داد كه در تحقيقات مبتنئي

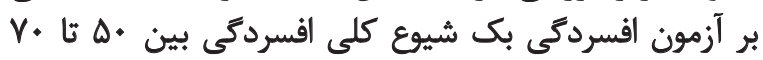

شيوع و شدت علائم افسردكى، اضطراب و افكار خودكشى به

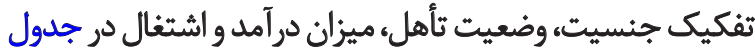
شماره ا نشان داده شده است.

بررسى ارتباط بين دادهاى جمعيتشناختى و آزمايشعاهى با

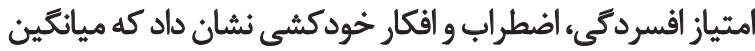
امتياز آزمون اضطراب بك ثناوت معنادارى بين دو كروه شاغل

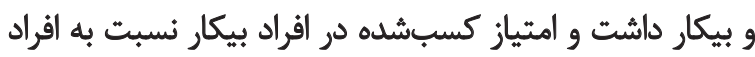

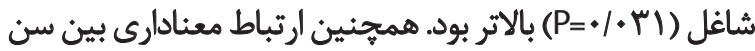

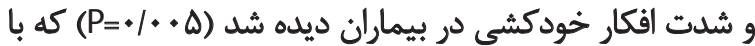

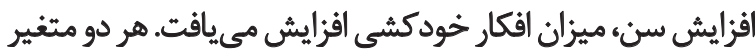
ارتباط معكوس و معنادارى با شدات افسردكي إنى

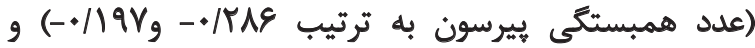

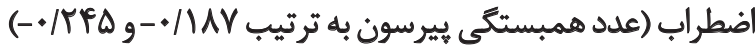

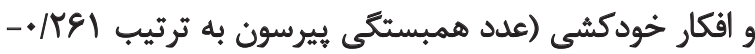

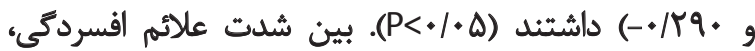

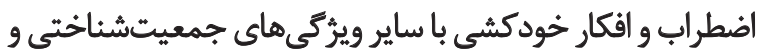


هراكه آزمونهاى مختلف نقطه برش متفاوتى براى تشخيص

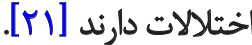

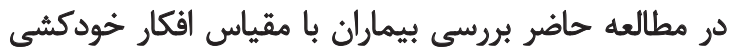

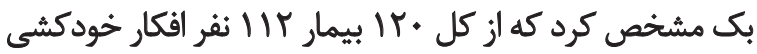

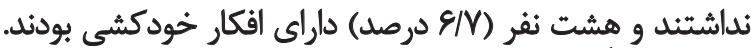

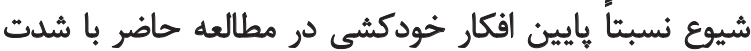
افسردگى خفيف تا متوسط در بيماران همخوانى دارئ دارد. مطالعات

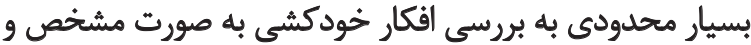

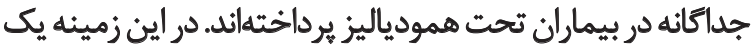

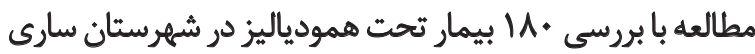

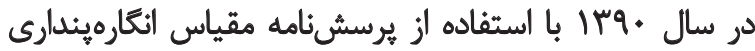

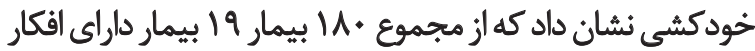

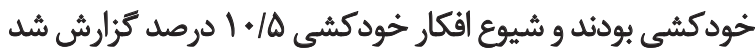

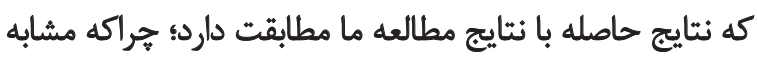

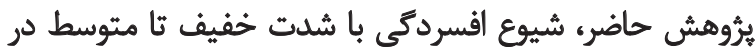

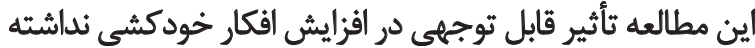

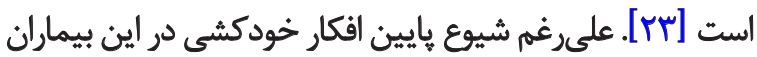

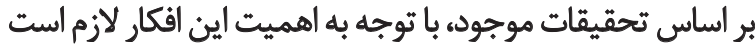
كه بررسى هاى بيشترى در اين زمينه انجام شود.

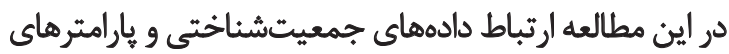

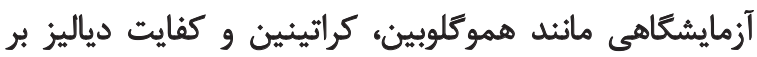

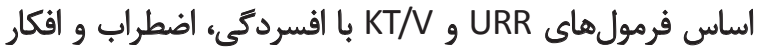

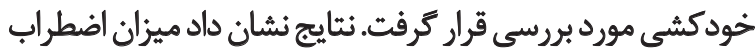

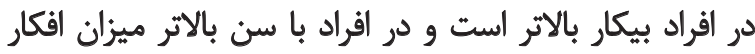

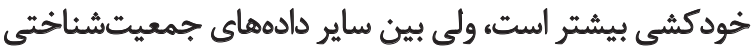

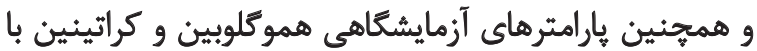

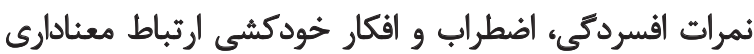
وجود نداشت.

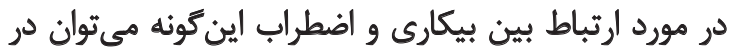

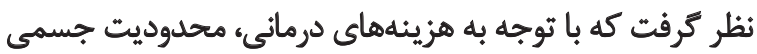

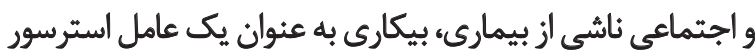

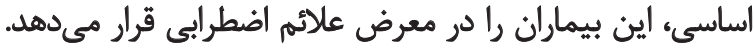

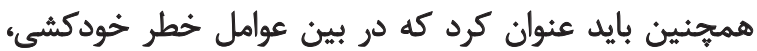

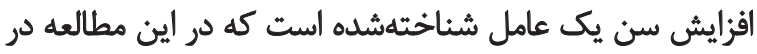

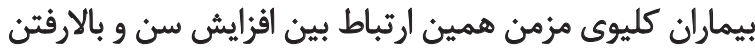

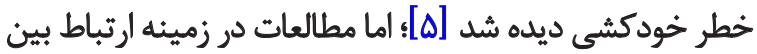

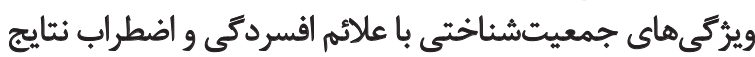

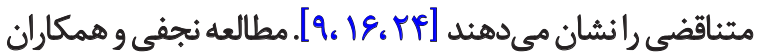

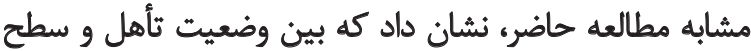

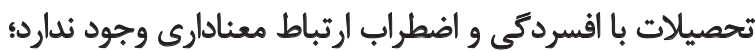

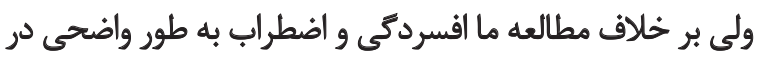

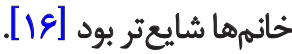

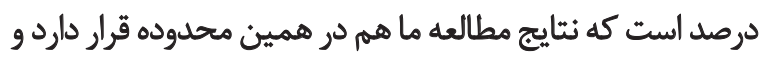

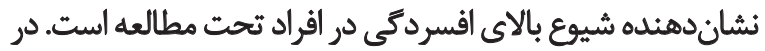

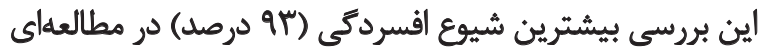

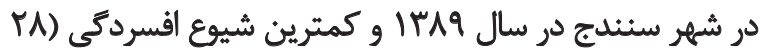

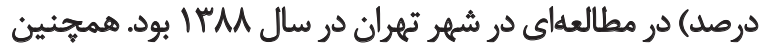

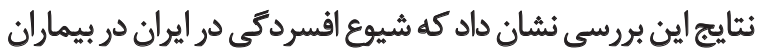

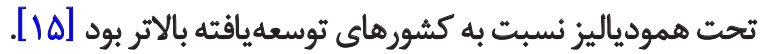

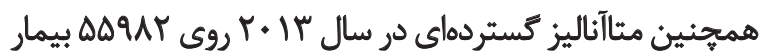

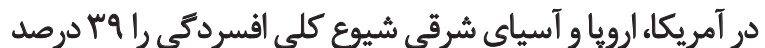

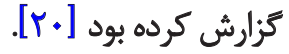

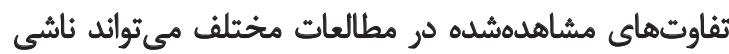

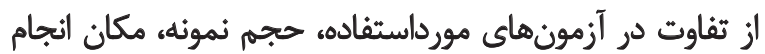

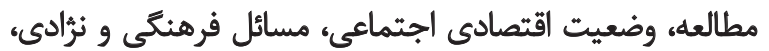

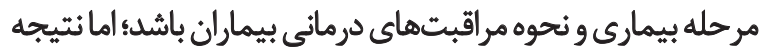

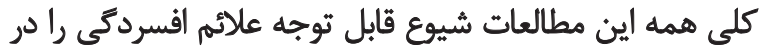

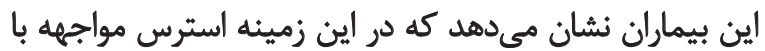

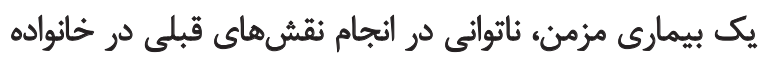

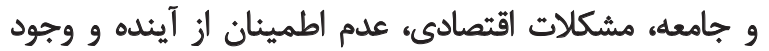

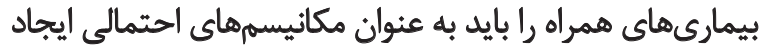

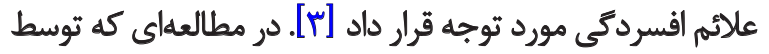

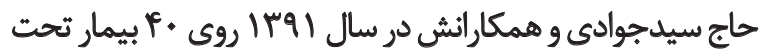

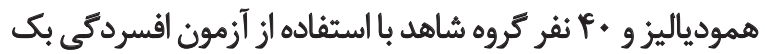

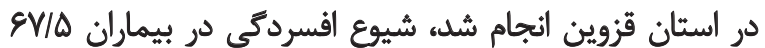

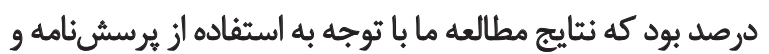

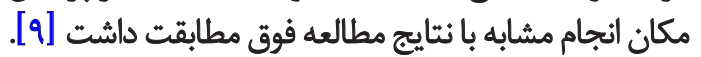
شيوع اضطراب در جامعه هدف مابر اساس آزمون اضطراب بك بئ

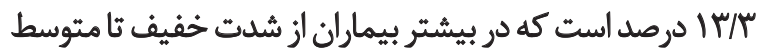

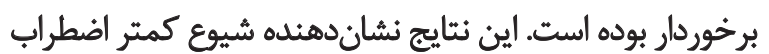
نسبت به افسردگى در اين مطالعه است. در مقايسه با با مطالعات

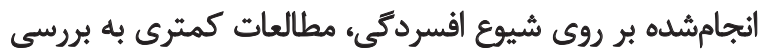

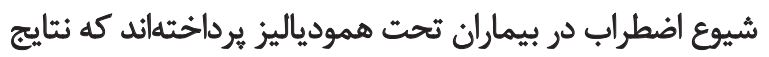

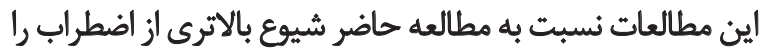

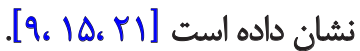

مطالعه نجفى و همكاران در سه واحد دياليز در شهر تهران

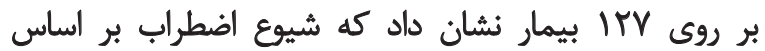

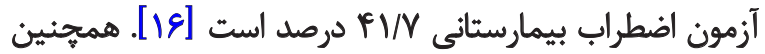

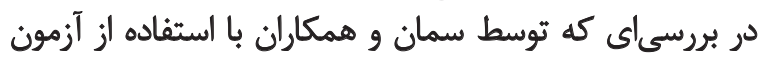

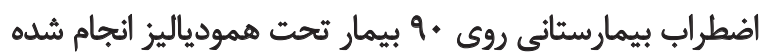

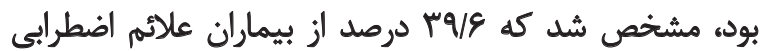

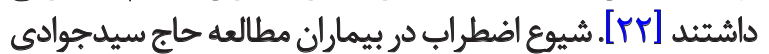

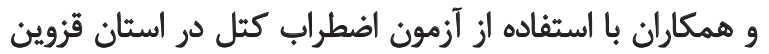

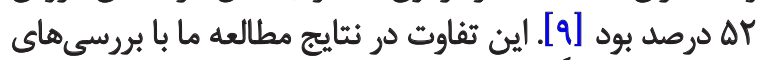

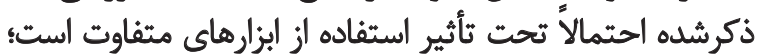


بنابراين ارزيابى علائم روانيزشكى بهخصوص افسردگى و اضطراب

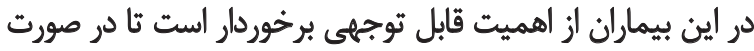

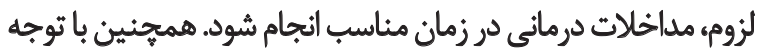

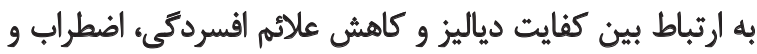

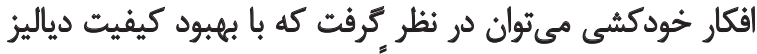

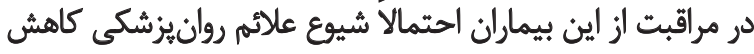

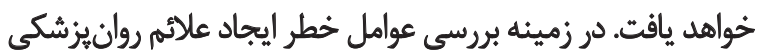

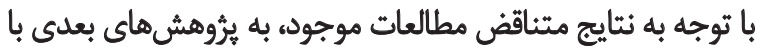

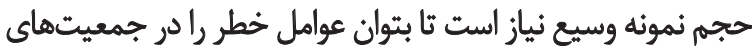
مختلف به صورت جداكانه مشخص كرد.

مالامظات اخغالقي

\section{يبروى ازٔ اصول اخلاق يثروهش}

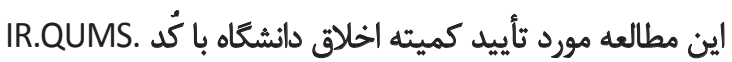

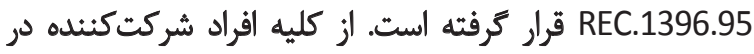
مطالعه رضايتنامه آكاهانه كرفته شد.

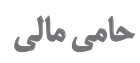

راين مقاله بر اساس بايانانامه خانم يريسا رحيمى در تروهي روانيزشكى دانشكاه علوميزشكى قزوين نوشته شده است.

$$
\text { مشاركت نويسندكان }
$$

روش شناسى: سيد حسين قافله باشى و محمد ابراهيم ساريجلو.

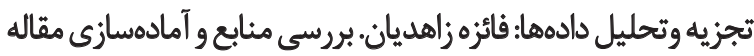
اوليه: يريسا رحيمى. ويراستارى و مديريت يروره: فائزه زاهديان.

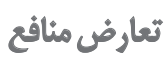

بنابر اظهار نويسندكان اين مقاله تعارض منافع ندارد.

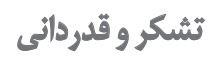

نويسندكان از معاونت يُورهشى دانشكاه علوميزشكى قزوين تشكر

$$
\text { و قدادانى هي كنيند. }
$$

مطالعه ناظميان و همكار ان نشان داد كه بين افسردتى و اضطراب

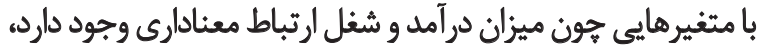

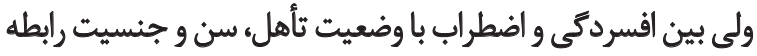

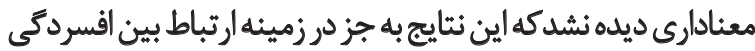

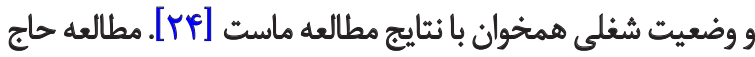

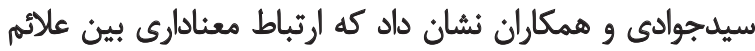

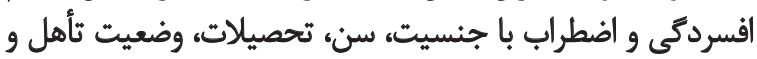

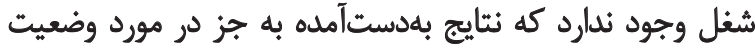

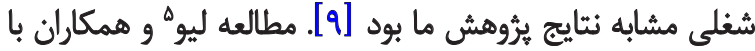

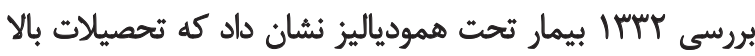

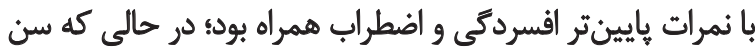

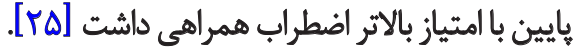

به نظر مي آيد عوامل جمعيتشناختى در هر منطقه ميتوانئد

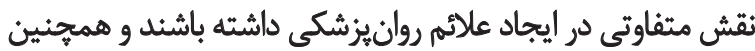

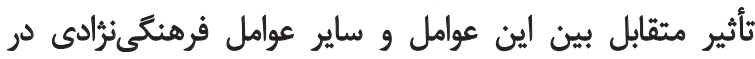

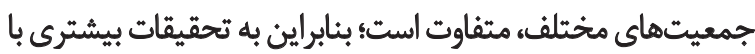

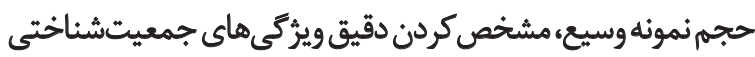

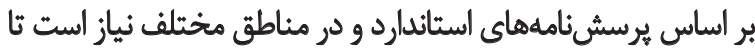

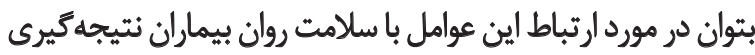

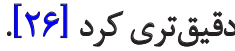

بر اساس مطالعه ما، بين كفايت دياليز بانمرات افسردگى اضطراب

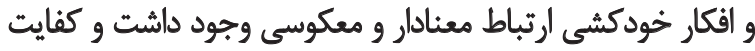

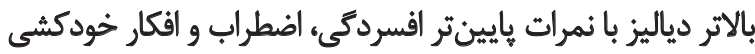

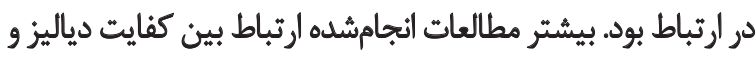

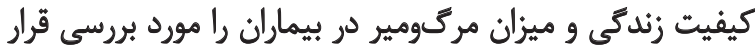

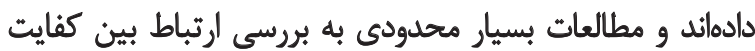

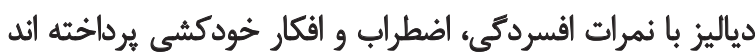

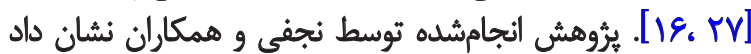

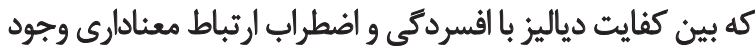

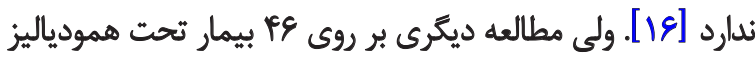

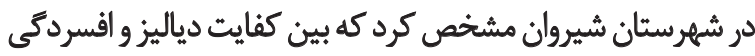
ارتباط معكوس و معناداري وجود داشت [بردئ. ارتباط معكوس و معنادار بين كفايت دياليز با ميزان افسردگى فئ،

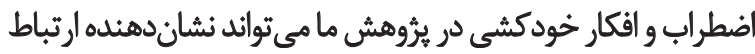

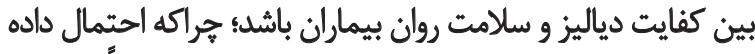

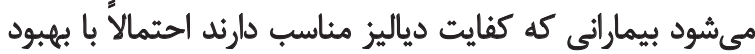

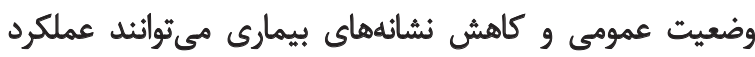

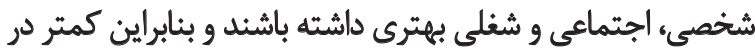

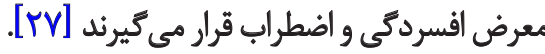

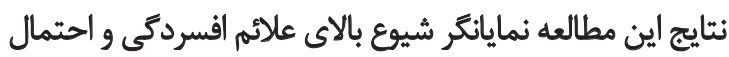
وجود علائم اضطر ابى در بيماران كليوى مزمن تحت همودياليز است؛ 


\section{References}

[1] El Filali A, Bentata Y, Ada N, Oneib B. Depression and anxiety disorders in chronic hemodialysis patients and their quality of life: A cross-sectional study about 106 cases in the Northeast of Morocco. Saudi J Kidney Dis Transpl. 2017; 28(2):341-5. [DOI:10.4103/1319-2442.202785] [PMID]

[2] Teles F, de Azevedo VFD, de Miranda CT, de Melo Miranda MP, do Carmo Teixeira M, Elias RM. Depression in hemodialysis patients: The role of dialysis shift. Clinics. 2014; 69(3):198-202. [DOI:10.6061/clinics/2014(03)10] [PMID] [PMCID]

[3] Palmer S, Vecchio M, Craig JC, Tonelli M, Johnson DW, Nicolucci $A$, et al. Prevalence of depression in chronic kidney disease: Systematic review and meta-analysis of observational studies. Kidney Int. 2013; 84(1):179-91. [DOI:10.1038/ki.2013.77] [PMID]

[4] Hajseyed Javadi AR, Ziaee A, Yazdi Z, Ebrahimabadi N, Shfikhani AA. Prevalence of anxiety and depression in diabetic patients: A comparative study. Biotech Health Sci. 2017:e41629. [DOI:10.5812/bhs.41629]

[5] Abbasi M, Yazdi Z, Farrokh Z, Hajseyed Javadi AR. Association of depression and anxiety with osteoarthritis. J Qazvin Univ Med Sci. 2014; 18(4):28-34. [In Persian] http://eprints.qums. ac.ir/772/

[6] Hajseyed Javadi AR, Shafikhani AA. Evaluation of depression and anxiety in patients with thalassemia: A comparative study. J Qazvin Univ Med Sci. 2017; 21(2):13-21. [In Persian] http:// eprints.qums.ac.ir/7354/

[7] dos Santos Pereira B, da Silva Fernandes N, de Melo NP, Abrita R, dos Santos Grincenkov FR, da Silva Fernandes NM. Beyond quality of life: A cross sectional study on the mental health of patients with chronic kidney disease undergoing dialysis and their caregivers. Health Qual Life Outcomes. 2017; 15:74. [DOI:10.1186/s12955-017-0646-4] [PMID] [PMCID]

[8] Hawamdeh S, Almari AM, Almutairi AS, Dator WL. Determinants and prevalence of depression in patients with chronic renal disease and their caregivers. Int J Nephrol Renovasc Dis. 2017; 10:183-9. [DOI:10.2147/IJNRD.S139652] [PMID] [PMCID]

[9] Hajseyed Javadi AR, Shafikhani AA, Allami A, Charkhchian $M$, Shiraghaee $M$. Depression and anxiety in chronic kidney disease patients on hemodialysis. Int J Appl Behav Sci. 2017; 4(1):21-7. http://journals.sbmu.ac.ir/ijabs/article/view/14669

[10] Alqarni AM, Alghamdi EA, Alaqil NA, Alzahrani AH, Aldhfyan YM, Alruwaili SA. Prevalence of anxiety and depression and its related influencing factors among Patients with end-stage renal disease on hemodialysis in Al-Kharj, Saudi Arabia. Int J Med Res Health Sci. 2019; 8(1):55-62. https://www.ijmrhs. com/abstract/prevalence-of-anxiety-and-depression-and-itsrelated-influencing-factors-among-patients-with-endstagerenal-disease-on-h-15502.html

[11] Gerogianni G, Polikandrioti M, Babatsikou F, Zyga S, Alikari V, Vasilopoulos G, et al. Anxiety-depression of dialysis patients and their caregivers. Medicina. 2019; 55(5):168. [DOI:10.3390/ medicina55050168] [PMID] [PMCID]

[12] Shirazian Sh, Grant CD, Aina O, Mattana J, Khorassani F, Ricardo AC. Depression in chronic kidney disease and end-stage renal disease: Similarities and differences in diagnosis, epidemiology and management. Kidney Int Rep. 2017; 2(1):94-107. [DOI:10.1016/j.ekir.2016.09.005] [PMID] [PMCID]

[13] Farrokhi F, Abedi N, Beyene J, Kurdyak P, Jassal SV. Association between depression and mortality in patients receiving longterm dialysis: A systematic review and meta-analysis. Am J Kidney Dis. 2014; 63(4):623-35. [DOI:10.1053/j.ajkd.2013.08.024] [PMID]

[14] Susan Hedayati S, Yalamanchili V, Finkelstein FO. A practical approach to the treatment of depression in patients with chronic kidney disease and end-stage renal disease. Kidney Int. 2012; 81(3):247-55. [DOI:10.1038/ki.2011.358] [PMID] [PMCID]

[15] Mirzaei M, Akbari Z. Prevalence of depression in dialysis patients in Iran (1998-2013): A systematic review and meta-analysis. J Mazandaran Univ Med Sci. 2015; 24(121):317-25. [In Persian] http://jmums.mazums.ac.ir/article-1-5191-en.html

[16] Najafi A, Keihani S, Bagheri N, Ghanbari Jolfaei A, Mazaheri Meybodi A. Association between anxiety and depression with dialysis adequacy in patients on maintenance hemodialysis. Iran J Psychiatry Behav Sci. 2016; 10(2):e4962. [DOI:10.17795/ ijpbs-4962] [PMID] [PMCID]

[17] Ghassemzadeh H, Mojtabai R, Karamghadiri N, Ebrahimkhani $\mathrm{N}$. Psychometric properties of a Persian-language version of the Beck Depression Inventory-second edition: BDI-II-Persian. Depress Anxiety. 2005; 21(4):185-92. [DOI:10.1002/da.20070] [PMID]

[18] Kaviani H, Mousavi AS. Psychometric properties of the Persian version of Beck anxiety inventory (BAI). Tehran Univ Med J. 2008; 66(2):136-40. http://tumj.tums.ac.ir/article-1-641-en. html

[19] Esfahani M, Hashemi Y, Alavi K. Psychometric assessment of Beck scale for suicidal ideation (BSSI) in general population in Tehran. Med J Islam Repub Iran. 2015; 29:268. http://mjiri. iums.ac.ir/article-1-3204-en.html

[20] Rajan EJE, Subramanian S. The effect of depression and anxiety on the performance status of end-stage renal disease patients undergoing hemodialysis. Saudi J Kidney Dis Transpl. 2016; 27(2):331-4. [DOI:10.4103/1319-2442.178555] [PMID]

[21] Ma TKW, Li PKT. Depression in dialysis patients. Nephrology. 2016; 21(8):639-46. [DOI:10.1111/nep.12742] [PMID]

[22] Semaan V, Noureddine S, Farhood L. Prevalence of depression and anxiety in end-stage renal disease: A survey of patients undergoing hemodialysis. Appl Nurs Res. 2018; 43:80-5. [DOI:10.1016/j.apnr.2018.07.009] [PMID]

[23] Spahbodi F, Hosseini SH, Makhloogh A, Sadeghie O, Taghipoor $M$. The suicide ideations rate and its risk factors among patients under hemodialysis. J Mazandaran Univ Med Sci. 2014; 23(110):110-6. [In Persian] http://jmums.mazums. ac.ir/article-1-3398-en.html

[24] Nazemian F, Ghafari F, Poorghaznein T. Evaluation of depression and anxiety in hemodialysis patients. Med J Mashhad Univ Med Sci. 2008; 51(3):171-6. [In Persian] http://eprints. mums.ac.ir/7713/ 
[25] Liu WJ, Musa R, Chew TF, Lim CTS, Morad Z, Adam bin Bujang M. DASS21: A useful tool in the psychological profile evaluation of dialysis patients. Am J Med Sci. 2018; 355(4):322-30. [DOI:10.1016/j.amjms.2017.11.015] [PMID]

[26] Vasilopoulou C, Bourtsi E, Giaple S, Koutelekos I, Theofilou P, Polikandrioti $\mathrm{M}$. The impact of anxiety and depression on the quality of life of hemodialysis patients. Glob J Health Sci. 2015; 8(1):45-55. [DOI:10.5539/gjhs.v8n1p45] [PMID] [PMCID]

[27] Hashemi M, Garshad A, Mirzaei SH, Kazemi F. Relationship between adequacy of dialysis and depression in hemodialysis patients in Imam Khomeini Hospital of Shirvan. J North Khorasan Univ Med Sci. 2014; 5(4):861-6. [In Persian] [DOI:10.29252/jnkums.5.4.861] 
This Page Intentionally Left Blank 